\title{
Vibrational Spectroscopic Study of (E)-4-(Benzylideneamino)-N-Carbamimidoyl Benzenesulfonamide
}

\author{
Asha Chandran, ${ }^{1}$ Sheena Mary, ${ }^{2}$ Hema Tresa Varghese, ${ }^{2}$ C. Yohannan Panicker, ${ }^{3,4}$ \\ T. K. Manojkumar, ${ }^{5}$ Christian Van Alsenoy, ${ }^{6}$ and G. Rajendran ${ }^{7}$ \\ ${ }^{1}$ Department of Chemistry, TKM College of Arts and Science, Kollam 695 005, Kerala, India \\ ${ }^{2}$ Department of Physics, Fatima Mata National College, Kollam 695 001, India \\ ${ }^{3}$ Department of Physics, TKM College of Arts and Science, Kollam 695 005, India \\ ${ }^{4}$ Department of Physics, Research Centre, Mar Ivanios College, Nalanchira, Thiruvananthapuram 695015, India \\ ${ }^{5}$ Indian Institute of Information Technology and Management-Kerala, Technopark, Thiruvananthapuram 695581, India \\ ${ }^{6}$ Department of Chemistry, University of Antwerp, 2610 Antwerp, Belgium \\ ${ }^{7}$ Department of Chemistry, University College Thiruvananthapuram, Thiruvananthapuram 695034, India
}

Correspondence should be addressed to C. Yohannan Panicker, cyphyp@rediffmail.com

Received 8 September 2011; Accepted 13 October 2011

Academic Editors: S. E. Jorge-Villar and A. Taga

Copyright ( $) 2012$ Asha Chandran et al. This is an open access article distributed under the Creative Commons Attribution License, which permits unrestricted use, distribution, and reproduction in any medium, provided the original work is properly cited.

The Fourier transform infrared and Fourier transform Raman spectra of (E)-4-(benzylideneamino)-N-carbamimidoyl benzenesulfonamide were recorded and analyzed. Geometry and harmonic vibrational wavenumbers were calculated theoretically using Gaussian 03 set of quantum chemistry codes. Calculations were performed at the Hartree-Fock (HF) and density functional theory (DFT; B3PW91, B3LYP) levels of theory. The calculated wavenumbers (B3LYP) agree well with the observed wavenumbers. Potential energy distribution is done using GAR2PED program. The red shift of the N-H stretching band in the infrared spectrum from the computed wavenumber indicates the weakening of the N-H bond. The geometrical parameters of the title compound are in agreement with that of reported similar derivatives. The calculated first hyperpolarizability is comparable with the reported value of similar derivative and may be an attractive object for further studies of nonlinear optics. Potential energy surface scan studies have been carried out to understand the stability of planar and nonplanar structures of the molecule.

\section{Introduction}

Benzenesulfonamide derivatives find wide applications for the synthesis of pharmaceutical products which have bactericidal properties and of various bioactive agents, artificial fibers, dyes, and plasticizers, and the synthesis of high molecular weight substances. Sulfonamides are chemotherapeutics most commonly used in veterinary practices because of their inexpensiveness and wide spectrum antimicrobial activity [1]. Sulfonamides represent one of the classical chemotypes associated with potent CA inhibition [2-5]. The chemistry of sulfonamides has been known as synthons in the preparation of various valuable biologically active compounds $[6,7]$ used as an antibacterial [8], protease inhibitor [9], diuretic [10], antitumor [11], and hypoglycaemic [12]. Singh et al. [13] reported the synthesis and antimicrobial activity of Schiff's and N-Mannich bases of Isatin and its derivatives with 4amino-N-carbamimidoyl benzene sulfonamide. There has been growing interest in using organic materials for nonlinear optical (NLO) devices, functioning as second harmonic generators, frequency converters, electro-optical modulators, and so forth because of the large second order electric susceptibilities of organic materials. The organic compound showing high hyperpolarizability are those containing an electron-donating group and an electron-withdrawing group interacting through a system of conjugated double bonds. In the case of sulfonamides, the electron-withdrawing group is the sulfonyl group $[14,15]$. To our knowledge, no theoretical 


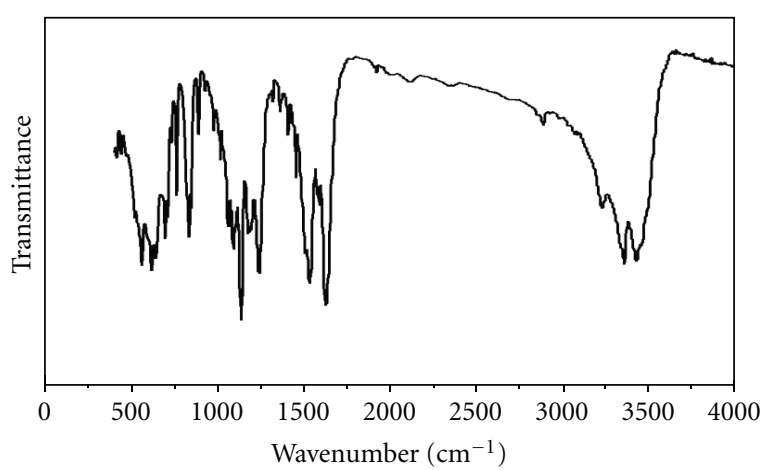

Figure 1: FT-IR spectrum of (E)-4-(benzylideneamino)-N-carbamimidoyl benzenesulfonamide.

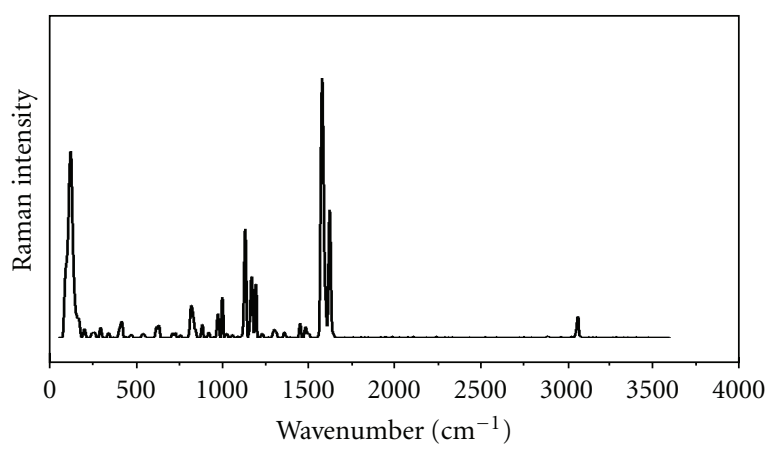

FIGURE 2: FT-Raman spectrum of (E)-4-(benzylideneamino)-Ncarbamimidoyl benzenesulfonamide.

HF or density functional theory (DFT) calculations or detailed vibrational infrared or Raman analyses have been performed on the title compound.

\section{Experimental}

All the chemicals were procured from Sigma-Aldrich, USA. $0.5 \mathrm{mg}$ of sulphaguanidine and $0.3 \mathrm{~mL}$ of benzaldehyde in $20 \mathrm{~mL}$ ethyl alcohol are refluxed for $3 \mathrm{hrs}$. The white precipitate was filtered off, washed with ethanol, and dried. Elemental analysis: found/calculated (\%): C 55.50/55.63; H 4.59/4.63; N 18.40/18.50; S 10.58/10.59. The FT-IR spectrum (Figure 1) was recorded on a DR/Jasco FT-IR 6300 spectrometer in $\mathrm{KBr}$ pellets, number of scans 16 , resolution $2 \mathrm{~cm}^{-1}$. The FT-Raman spectrum (Figure 2) was obtained on a BRUKER RFS 100/S, Germany. For excitation of the spectrum the emission of an Nd:YAG laser was used, excitation wavelength $1064 \mathrm{~nm}$, maximal power $150 \mathrm{~mW}$, measurement of solid sample. One thousand scans were accumulated with a total registration time of about $30 \mathrm{~min}$. The spectral resolution after apodization was $2 \mathrm{~cm}^{-1}$.

\section{Computational Details}

Calculations of the title compound were carried out with Gaussin 03 program [16] using the HF/6-31G*, B3PW91/6$31 \mathrm{G}^{*}$ and $\mathrm{B} 3 \mathrm{LYP} / 6-31 \mathrm{G}^{*}$, basis sets to predict the molecular structure and vibrational wavenumbers. The wavenumber values computed contain known systematic errors [17] and we, therefore, have used the scaling factor values of 0.8929 and 0.9613 for HF and DFT basis sets. Parameters corresponding to optimized geometry (B3LYP) of the title compound (Figure 3 ) are given in Table 1 . The absence of imaginary values of wavenumbers on the calculated vibrational spectrum confirms that the structure deduced corresponds to minimum energy. Potential energy distribution is done using GAR2PED program [18]. The assignment of the calculated wavenumbers is aided by the animation option of MOLEKEL program, which gives a visual presentation of the vibrational modes $[19,20]$. Potential energy surface scan studies have been carried out to understand the stability of planar and non planar structures of the molecule. The profiles of potential energy surface for torsion angles $\mathrm{C}_{15}-\mathrm{N}_{14}-$ $\mathrm{C}_{12}-\mathrm{C}_{3}, \mathrm{~N}_{28}-\mathrm{S}_{25}-\mathrm{C}_{22}-\mathrm{C}_{20}, \mathrm{~N}_{31}-\mathrm{C}_{30}-\mathrm{N}_{28}-\mathrm{S}_{25}$ and $\mathrm{N}_{32}-\mathrm{C}_{30}-$ $\mathrm{N}_{28}-\mathrm{S}_{25}$ are given in Figures 4-7. The energy is minimum for -177.0 ( -1309.42093 Hartree), 86.0 ( -1309.41690 Hartree), 12.6 (-1309.42098 Hartree), and $-169.9(-1309.42070$ Hartree) for the above torsion angles.

\section{Results and Discussion}

4.1. IR and Raman Spectra. The observed IR, Raman bands, and calculated (scaled) wavenumbers, and assignments are given in Table 2.

The asymmetric and symmetric stretching modes of $\mathrm{SO}_{2}$ group appear in the region $1360-1310$ and $1165-1135 \mathrm{~cm}^{-1}$ [21]. The observed bands at $1358,1130 \mathrm{~cm}^{-1}$ in the IR spectrum, $1360,1133 \mathrm{~cm}^{-1}$ in the Raman spectrum, and 1371 , $1148 \mathrm{~cm}^{-1}$ theoretically are assigned as $\mathrm{SO}_{2}$ stretching modes. These modes are not pure, but contain significant contributions from other modes also. Although the region of $\mathrm{SO}_{2}$ scissors $\left(560 \pm 40 \mathrm{~cm}^{-1}\right)$ and that of $\mathrm{SO}_{2}$ wagging $(500 \pm$ $55 \mathrm{~cm}^{-1}$ ) partly overlap, the two vibrations appear separately [21]. These deformation bands of $\mathrm{SO}_{2}$ are assigned at $515,469 \mathrm{~cm}^{-1}$ in the IR spectrum and at $510,457 \mathrm{~cm}^{-1}$ theoretically. Chohan et al. [22] reported the $\mathrm{SO}_{2}$ stretching vibrations at $1345,1110 \mathrm{~cm}^{-1}$ and $\mathrm{SN}$ and CS stretching modes at $833 \mathrm{~cm}^{-1}$ for sulfonamide derivatives. Hangen et al. [23] reported $\mathrm{SO}_{2}$ modes at 1314, 1308, 1274, 1157, 1147, and $1133 \mathrm{~cm}^{-1}$ and $\mathrm{SN}$ stretching modes at $917,920,932$, and $948 \mathrm{~cm}^{-1}$ for sulfonamide derivatives. The twisting mode of $\mathrm{SO}_{2}$ is expected at around $350 \mathrm{~cm}^{-1}$ [21]. The calculated bands (B3LYP) at $365,298 \mathrm{~cm}^{-1}$ are assigned as the twisting and rocking modes of $\mathrm{SO}_{2}$. The NS stretching vibration is expected [21] in the region $905 \pm 30 \mathrm{~cm}^{-1}$ and the band observed at $841 \mathrm{~cm}^{-1}$ in the IR spectrum and at $831 \mathrm{~cm}^{-1}$ theoretically are assigned as $v \mathrm{SN}$. The C-S stretching vibration is assigned at $692 \mathrm{~cm}^{-1}$ (IR) and at $679 \mathrm{~cm}^{-1}$ (DFT) $\mathrm{cm}^{-1}[21]$.

The $\mathrm{C}=\mathrm{N}$ stretching bands [24] are observed in the region $1550-1700 \mathrm{~cm}^{-1}$ and for the title compound, the B3LYP calculation give these bands at $1687\left(\mathrm{C}_{12}-\mathrm{N}_{14}\right), 1641 \mathrm{~cm}^{-1}\left(\mathrm{C}_{30}-\right.$ $\mathrm{N}_{31}$ ). The $\mathrm{CN}$ stretching vibrations [21] are moderately to strongly active in the region $1275 \pm 55 \mathrm{~cm}^{-1}$. Primary aromatic amines with nitrogen directly on the ring absorb at $1330-1200 \mathrm{~cm}^{-1}$ because of the stretching of the phenyl 


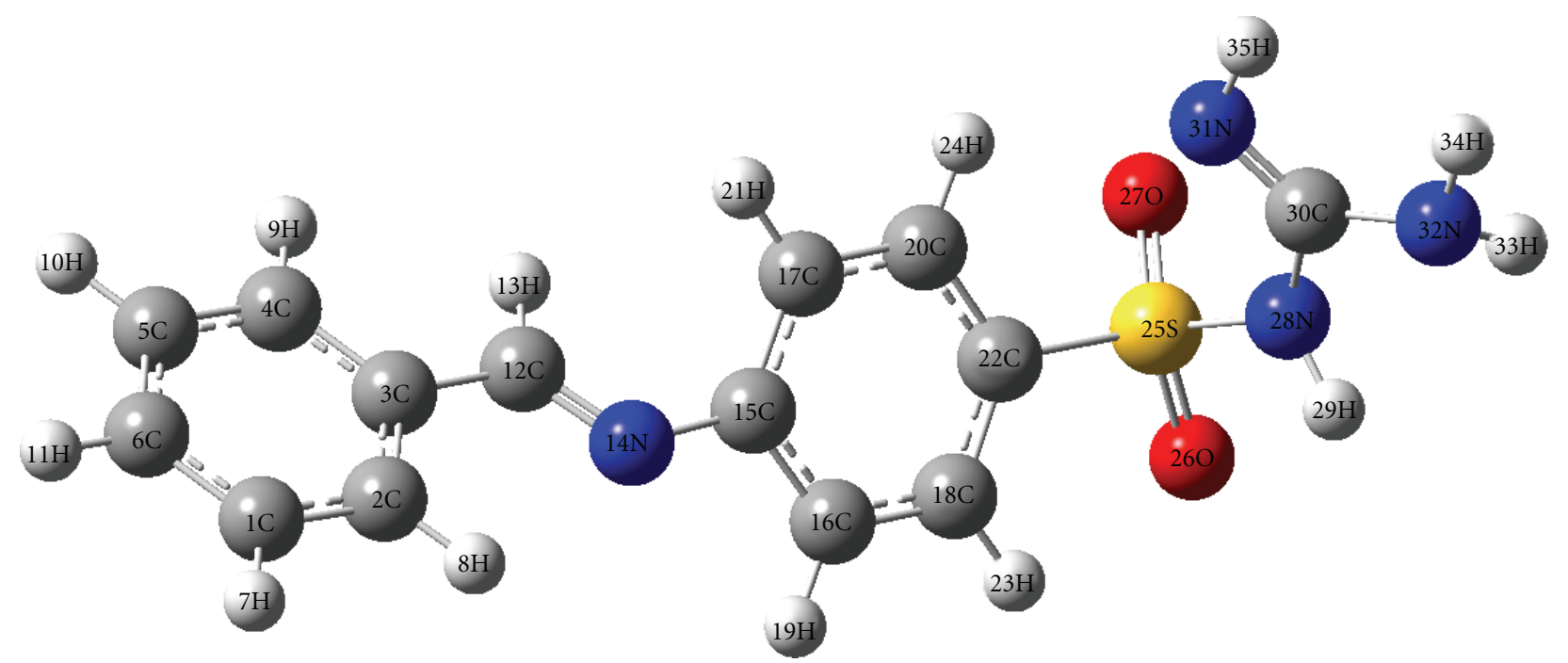

FIGURE 3: Optimized geometry (B3LYP) of (E)-4-(benzylideneamino)-N-carbamimidoyl benzene sulfonamide.

carbon nitrogen bond [25]. For the title compound $\mathrm{C}_{15}-$ $\mathrm{N}_{14}$ stretching mode is observed at $1281 \mathrm{~cm}^{-1}$ theoretically. Panicker et al. [26] reported the $\mathrm{CN}$ stretching modes at $1219,1237 \mathrm{~cm}^{-1}$ (IR), $1222 \mathrm{~cm}^{-1}$ (Raman) and at 1292, 1234, $1200 \mathrm{~cm}^{-1}$ theoretically. The $\mathrm{CN}$ stretching modes $\mathrm{C}_{30}-\mathrm{N}_{28}$ and $\mathrm{C}_{30}-\mathrm{N}_{32}$ are assigned at 1100 and $942 \mathrm{~cm}^{-1}$ theoretically which is expected in the range $950-1115 \mathrm{~cm}^{-1}$ [21].

The $\mathrm{NH}_{2}$ stretching modes are expected in the region $3260-3390 \mathrm{~cm}^{-1}$ [21] and in the present case the bands observed at $3421 \mathrm{~cm}^{-1}$ in the IR spectrum and bands at 3501 , $3399 \mathrm{~cm}^{-1}$ (DFT) are assigned as $\mathrm{NH}_{2}$ stretching modes. The bands corresponding to $\delta \mathrm{NH}_{2}$ vibrations are expected in the region $1610 \pm 30 \mathrm{~cm}^{-1}$ [21]. In the present case, the band at $1620 \mathrm{~cm}^{-1}$ (IR), $1625 \mathrm{~cm}^{-1}$ (Raman) and $1602 \mathrm{~cm}^{-1}$ (DFT) is assigned as $\delta \mathrm{NH}_{2}$. The rocking/twisting mode of $\mathrm{NH}_{2}$ is expected in the region $1195 \pm 90 \mathrm{~cm}^{-1}$ and this mode is assigned at $1182 \mathrm{~cm}^{-1}$ in IR, $1188 \mathrm{~cm}^{-1}$ in Raman spectrum at 1181 (DFT) $\mathrm{cm}^{-1}$. The wagging mode of $\mathrm{NH}_{2}$ is assigned at $778 \mathrm{~cm}^{-1}$ theoretically which is expected in the region $840 \pm 55 \mathrm{~cm}^{-1}$ [21]. The torsional $\mathrm{NH}_{2}$ mode is expected below $400 \mathrm{~cm}^{-1}$ [21] and these modes are identified and assigned (Table 2). For sulfonamide derivatives the $\mathrm{NH}_{2}$ modes are reported at 3390, 3395, at $3399 \mathrm{~cm}^{-1}$ [22].

The NH stretching vibrations generally give rise to bands at $3500-3300 \mathrm{~cm}^{-1}[27,28]$. In the present study, the $\mathrm{N}_{28}-\mathrm{H}_{29}$ stretching bands have split into a doublet, 3351, $3222 \mathrm{~cm}^{-1}$ in the IR spectrum owing to the Davydov coupling between neighboring units. The splitting of about $129 \mathrm{~cm}^{-1}$ in the IR spectrum is due to the strong intermolecular hydrogen bonding. Furthermore the NH stretching wave number is red shifted by $129 \mathrm{~cm}^{-1}$ in the IR spectrum with a strong intensity from the computed wavenumber which indicate the weakening of the $\mathrm{NH}$ bond [29]. The DFT calculations give the $\mathrm{N}_{31}-\mathrm{H}_{35}$ stretching mode at $3453 \mathrm{~cm}^{-1}$. In $\mathrm{N}$-mono substituted amides, the in plane bend ing frequency and the resonance stiffened $\mathrm{CN}$ bands stretching frequency fall close together and therefore interact. The NH deformation band of guanidine structural motif is expected in the region $1395 \pm 25 \mathrm{~cm}^{-1}[21,25]$. The DFT calculations give these modes at 1442 and $1485 \mathrm{~cm}^{-1}$. The bands observed at $1500,1450 \mathrm{~cm}^{-1}$ in the IR spectrum and at $1486,1446 \mathrm{~cm}^{-1}$ in the Raman spectrum are assigned as the deformation bands of $\mathrm{NH}$ group. The out-of-plane $\mathrm{NH}$ deformation is expected in the region $650 \pm 50 \mathrm{~cm}^{-1}$ [21] and bands at 727 (IR), 729 (Raman) and 706, $608 \mathrm{~cm}^{-1}$ (DFT) are assigned as these modes.

Since the identification of all the normal modes of vibration of large molecules is not trivial, we tried to simplify the problem by considering each molecule as a substituted benzene. Such an idea has already been successfully utilized by several workers for the vibrational assignments of molecules containing homo- and heteroaromatic rings [30-32]. In the following discussion, mono- and parasubstituted phenyl rings are designated as $\mathrm{PhI}$ and PhII, respectively. The modes in the phenyl rings will differ in wavenumber and the magnitude of splitting will depend on the strength of the interactions between different parts (internal coordinates) of the two rings. For some modes, this splitting is so small that they may be considered as quasidegenerate, and for some other modes a significant amount of splitting is observed. Such observations have already been reported $[33,34]$.

The phenyl CH stretching modes occur above $3000 \mathrm{~cm}^{-1}$ and are typically exhibited as multiplicity of weak to moderate bands compared with the aliphatic $\mathrm{CH}$ stretching [35]. In the present case, the DFT calculations give $v \mathrm{CH}$ modes of the phenyl rings in the range $3132-3057 \mathrm{~cm}^{-1}$. The bands observed at $3026,3073 \mathrm{~cm}^{-1}$ in the IR spectrum and at $3066 \mathrm{~cm}^{-1}$ in the Raman spectrum were assigned as $\mathrm{CH}$ stretching modes of the phenyl rings.

The benzene ring possesses six ring stretching vibrations, of which the four with the highest wavenumbers (occurring near $1600,1580,1490$, and $1440 \mathrm{~cm}^{-1}$ ) are good group vibrations. With heavy substituents, the bands tend to shift to somewhat lower wavenumbers. In the absence of ring conjugation, the band at $1580 \mathrm{~cm}^{-1}$ is usually weaker than that at $1600 \mathrm{~cm}^{-1}$. In the case of $\mathrm{C}=\mathrm{O}$ substitution, the band 
TABLE 1: Geometrical (B3LYP) parameters of (E)-4-(benzylideneamino)-N-carbamimidoyl benzenesulfonamide, atom labeling according to Figure 3.

\begin{tabular}{|c|c|c|c|c|c|}
\hline \multicolumn{2}{|c|}{ Bond lengths $(\AA)$} & \multicolumn{2}{|c|}{ Bond angles $\left({ }^{\circ}\right)$} & \multicolumn{2}{|c|}{ Dihedral angles $\left({ }^{\circ}\right)$} \\
\hline $\mathrm{C}_{1}-\mathrm{C}_{2}$ & 1.3901 & $\mathrm{~A}(2,1,6)$ & 120.2 & $\mathrm{D}(6,1,2,3)$ & -0.0 \\
\hline $\mathrm{C}_{1}-\mathrm{C}_{6}$ & 1.4001 & $\mathrm{~A}(2,1,7)$ & 119.8 & $\mathrm{D}(6,1,2,8)$ & -180.0 \\
\hline $\mathrm{C}_{1}-\mathrm{H}_{7}$ & 1.0866 & $\mathrm{~A}(6,1,7)$ & 119.9 & $\mathrm{D}(7,1,2,3)$ & 180.0 \\
\hline $\mathrm{C}_{2}-\mathrm{C}_{3}$ & 1.4062 & $\mathrm{~A}(1,2,3)$ & 120.1 & $\mathrm{D}(7,1,2,8)$ & 0.0 \\
\hline $\mathrm{C}_{2}-\mathrm{H}_{8}$ & 1.0852 & $\mathrm{~A}(1,2,8)$ & 121.3 & $\mathrm{D}(2,1,6,5)$ & 0.1 \\
\hline $\mathrm{C}_{3}-\mathrm{C}_{4}$ & 1.4035 & $\mathrm{~A}(3,2,8)$ & 118.6 & $\mathrm{D}(2,1,6,11)$ & -180.0 \\
\hline $\mathrm{C}_{3}-\mathrm{C}_{12}$ & 1.4666 & $\mathrm{~A}(2,3,4)$ & 119.2 & $\mathrm{D}(7,1,6,5)$ & -180.0 \\
\hline $\mathrm{C}_{4}-\mathrm{C}_{5}$ & 1.3941 & $\mathrm{~A}(2,3,12)$ & 121.5 & $\mathrm{D}(7,1,6,11)$ & 0.0 \\
\hline $\mathrm{C}_{4}-\mathrm{H}_{9}$ & 1.0878 & $\mathrm{~A}(4,3,12)$ & 119.3 & $\mathrm{D}(1,2,3,4)$ & -0.0 \\
\hline $\mathrm{C}_{5}-\mathrm{C}_{6}$ & 1.3958 & $\mathrm{~A}(3,4,5)$ & 120.5 & $\mathrm{D}(1,2,3,12)$ & 180.0 \\
\hline $\mathrm{C}_{5}-\mathrm{H}_{10}$ & 1.0864 & $\mathrm{~A}(3,4,9)$ & 119.4 & $\mathrm{D}(8,2,3,4)$ & 179.9 \\
\hline $\mathrm{C}_{6}-\mathrm{H}_{11}$ & 1.0867 & $\mathrm{~A}(5,4,9)$ & 120.0 & $\mathrm{D}(8,2,3,12)$ & -0.2 \\
\hline $\mathrm{C}_{12}-\mathrm{H}_{13}$ & 1.1006 & $\mathrm{~A}(4,5,6)$ & 119.8 & $\mathrm{D}(2,3,4,5)$ & 0.1 \\
\hline $\mathrm{C}_{12}-\mathrm{N}_{14}$ & 1.2816 & $\mathrm{~A}(4,5,10)$ & 120.0 & $\mathrm{D}(2,3,4,9)$ & -179.9 \\
\hline $\mathrm{C}_{15}-\mathrm{N}_{14}$ & 1.4020 & $\mathrm{~A}(6,5,10)$ & 120.2 & $\mathrm{D}(12,3,4,5)$ & -179.8 \\
\hline $\mathrm{C}_{15}-\mathrm{C}_{16}$ & 1.4061 & $\mathrm{~A}(1,6,5)$ & 120.0 & $\mathrm{D}(12,3,4,9)$ & 0.2 \\
\hline $\mathrm{C}_{15}-\mathrm{C}_{17}$ & 1.4078 & $\mathrm{~A}(1,6,11)$ & 120.0 & $\mathrm{D}(2,3,12,13)$ & -179.7 \\
\hline $\mathrm{C}_{16}-\mathrm{C}_{18}$ & 1.3893 & $\mathrm{~A}(5,6,11)$ & 120.0 & $\mathrm{D}(2,3,12,14)$ & 1.1 \\
\hline $\mathrm{C}_{16}-\mathrm{H}_{19}$ & 1.0854 & $\mathrm{~A}(3,12,13)$ & 115.6 & $\mathrm{D}(4,3,12,13)$ & 0.2 \\
\hline $\mathrm{C}_{17}-\mathrm{C}_{20}$ & 1.3912 & $\mathrm{~A}(3,12,14)$ & 122.8 & $\mathrm{D}(4,3,12,14)$ & -179.0 \\
\hline $\mathrm{C}_{17}-\mathrm{H}_{21}$ & 1.0859 & $\mathrm{~A}(13,12,14)$ & 121.6 & $\mathrm{D}(3,4,5,6)$ & -0.1 \\
\hline $\mathrm{C}_{18}-\mathrm{C}_{22}$ & 1.3991 & $\mathrm{~A}(12,14,15)$ & 120.1 & $\mathrm{D}(3,4,5,10)$ & 180.0 \\
\hline $\mathrm{C}_{18}-\mathrm{H}_{23}$ & 1.0845 & $\mathrm{~A}(14,15,16)$ & 117.8 & $\mathrm{D}(9,4,5,6)$ & 180.0 \\
\hline $\mathrm{C}_{20}-\mathrm{C}_{22}$ & 1.3968 & $\mathrm{~A}(14,15,17)$ & 123.0 & $\mathrm{D}(9,4,5,10)$ & -0.0 \\
\hline $\mathrm{C}_{20}-\mathrm{H}_{24}$ & 1.0827 & $\mathrm{~A}(16,15,17)$ & 119.2 & $\mathrm{D}(4,5,6,1)$ & -0.0 \\
\hline $\mathrm{C}_{22}-\mathrm{S}_{25}$ & 1.7883 & $\mathrm{~A}(15,16,18)$ & 120.5 & $\mathrm{D}(4,5,6,11)$ & -180.0 \\
\hline $\mathrm{S}_{25}-\mathrm{O}_{26}$ & 1.4658 & $\mathrm{~A}(15,16,19)$ & 118.6 & $\mathrm{D}(10,5,6,1)$ & 180.0 \\
\hline $\mathrm{S}_{25}-\mathrm{O}_{27}$ & 1.4570 & $\mathrm{~A}(18,16,19)$ & 120.8 & $\mathrm{D}(10,5,6,11)$ & -0.0 \\
\hline $\mathrm{S}_{25}-\mathrm{N}_{28}$ & 1.7054 & $\mathrm{~A}(15,17,20)$ & 120.7 & $\mathrm{D}(3,12,14,15)$ & -177.0 \\
\hline $\mathrm{N}_{28}-\mathrm{H}_{29}$ & 1.0140 & $\mathrm{~A}(15,17,21)$ & 119.6 & $\mathrm{D}(13,12,14,15)$ & 3.8 \\
\hline $\mathrm{C}_{30}-\mathrm{N}_{28}$ & 1.4033 & $\mathrm{~A}(20,17,21)$ & 119.7 & $\mathrm{D}(12,14,15,16)$ & -140.4 \\
\hline $\mathrm{C}_{30}-\mathrm{N}_{31}$ & 1.2764 & $\mathrm{~A}(16,18,22)$ & 119.3 & $\mathrm{D}(12,14,15,17)$ & 42.1 \\
\hline $\mathrm{C}_{30}-\mathrm{N}_{32}$ & 1.3990 & $\mathrm{~A}(16,18,23)$ & 120.9 & $\mathrm{D}(14,15,16,18)$ & -179.6 \\
\hline $\mathrm{N}_{31}-\mathrm{H}_{35}$ & 1.0208 & $\mathrm{~A}(22,18,23)$ & 119.8 & $\mathrm{D}(14,15,16,19)$ & 1.7 \\
\hline $\mathrm{N}_{32}-\mathrm{H}_{33}$ & 1.0149 & $\mathrm{~A}(17,20,22)$ & 119.1 & $\mathrm{D}(17,15,16,18)$ & -2.1 \\
\hline $\mathrm{N}_{32}-\mathrm{H}_{34}$ & 1.0130 & $\mathrm{~A}(17,20,24)$ & 121.2 & $\mathrm{D}(17,15,16,19)$ & 179.2 \\
\hline - & - & $\mathrm{A}(22,20,24)$ & 119.7 & $\mathrm{D}(14,15,17,20)$ & 178.6 \\
\hline - & - & $\mathrm{A}(18,22,20)$ & 121.2 & $\mathrm{D}(14,15,17,21)$ & 0.6 \\
\hline - & - & $\mathrm{A}(18,22,25)$ & 118.7 & $\mathrm{D}(16,15,17,20)$ & 1.2 \\
\hline - & - & $\mathrm{A}(20,22,25)$ & 120.1 & $\mathrm{D}(16,15,17,21)$ & -176.8 \\
\hline - & - & $\mathrm{A}(22,25,26)$ & 107.7 & $\mathrm{D}(15,16,18,22)$ & 1.6 \\
\hline - & - & $\mathrm{A}(22,25,27)$ & 108.9 & $\mathrm{D}(15,16,18,23)$ & -177.7 \\
\hline - & - & $\mathrm{A}(22,25,28)$ & 107.7 & $\mathrm{D}(19,16,18,22)$ & -179.6 \\
\hline - & - & $\mathrm{A}(26,25,27)$ & 122.2 & $\mathrm{D}(19,16,18,23)$ & 1.0 \\
\hline - & - & $\mathrm{A}(26,25,28)$ & 101.5 & $\mathrm{D}(15,17,20,22)$ & -0.0 \\
\hline - & - & $\mathrm{A}(27,25,28)$ & 108.0 & $\mathrm{D}(15,17,20,24)$ & -178.6 \\
\hline - & - & $\mathrm{A}(25,28,29)$ & 112.2 & $\mathrm{D}(21,17,20,22)$ & 178.0 \\
\hline - & - & $\mathrm{A}(25,28,30)$ & 126.8 & $\mathrm{D}(21,17,20,24)$ & -0.5 \\
\hline - & - & $\mathrm{A}(25,28,30)$ & 117.8 & $\mathrm{D}(16,18,22,20)$ & -0.4 \\
\hline - & - & $\mathrm{A}(28,30,31)$ & 121.2 & $\mathrm{D}(16,18,22,25)$ & -179.4 \\
\hline
\end{tabular}


Table 1: Continued.

\begin{tabular}{|c|c|c|c|c|c|}
\hline \multicolumn{2}{|c|}{ Bond lengths $(\AA)$} & \multicolumn{2}{|c|}{ Bond angles $\left({ }^{\circ}\right)$} & \multicolumn{2}{|c|}{ Dihedral angles $\left({ }^{\circ}\right)$} \\
\hline - & - & $\mathrm{A}(28,30,32)$ & 110.2 & $\mathrm{D}(23,18,22,20)$ & 178.9 \\
\hline - & - & $\mathrm{A}(31,30,32)$ & 128.6 & $\mathrm{D}(23,18,22,25)$ & 0.0 \\
\hline- & - & $\mathrm{A}(30,31,35)$ & 111.1 & $\mathrm{D}(17,20,22,18)$ & -0.4 \\
\hline- & - & $\mathrm{A}(30,32,33)$ & 114.6 & $\mathrm{D}(17,20,22,25)$ & 178.5 \\
\hline - & - & $\mathrm{A}(30,32,34)$ & 113.9 & $\mathrm{D}(24,20,22,18)$ & 178.2 \\
\hline - & - & $\mathrm{A}(33,32,34)$ & 111.9 & $\mathrm{D}(24,20,22,25)$ & -2.9 \\
\hline- & - & - & - & $\mathrm{D}(18,22,25,26)$ & 13.7 \\
\hline- & - & - & - & $\mathrm{D}(18,22,25,27)$ & 148.1 \\
\hline- & - & - & - & $\mathrm{D}(18,22,25,28)$ & -95.1 \\
\hline- & - & - & - & $\mathrm{D}(20,22,25,26)$ & -165.2 \\
\hline - & - & - & - & $\mathrm{D}(20,22,25,27)$ & -30.8 \\
\hline- & - & - & - & $\mathrm{D}(20,22,25,28)$ & 86.0 \\
\hline- & - & - & - & $\mathrm{D}(22,25,28,29)$ & 91.5 \\
\hline- & - & - & - & $\mathrm{D}(22,25,28,30)$ & -67.8 \\
\hline- & - & - & - & $\mathrm{D}(26,25,28,29)$ & -21.4 \\
\hline- & - & - & - & $\mathrm{D}(26,25,28,30)$ & 179.3 \\
\hline- & - & - & - & $\mathrm{D}(27,25,28,29)$ & -151.1 \\
\hline- & - & - & - & $\mathrm{D}(27,25,28,30)$ & 49.6 \\
\hline - & - & - & - & $\mathrm{D}(25,28,30,31)$ & 12.6 \\
\hline- & - & - & - & $\mathrm{D}(25,28,30,32)$ & -169.9 \\
\hline- & - & - & - & $\mathrm{D}(29,28,30,31)$ & -145.7 \\
\hline- & - & - & - & $\mathrm{D}(29,28,30,32)$ & 31.8 \\
\hline- & - & - & - & $\mathrm{D}(28,30,31,35)$ & -173.1 \\
\hline- & - & - & - & $\mathrm{D}(32,30,31,35)$ & 9.8 \\
\hline- & - & - & - & $\mathrm{D}(28,30,32,33)$ & 43.7 \\
\hline- & - & - & - & $\mathrm{D}(28,30,32,34)$ & 174.5 \\
\hline- & - & - & - & $\mathrm{D}(31,30,32,33)$ & -139.0 \\
\hline- & - & - & - & $\mathrm{D}(31,30,32,34)$ & -8.2 \\
\hline
\end{tabular}

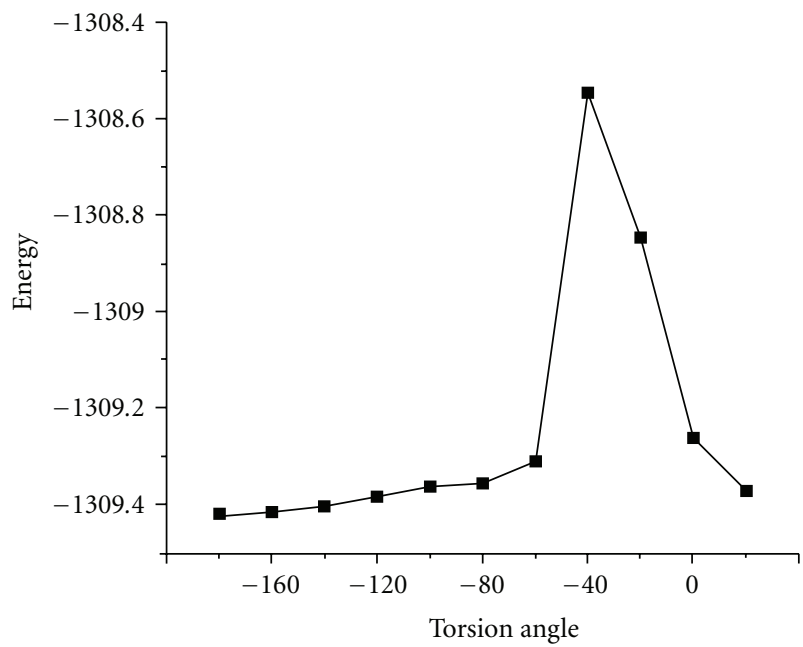

Figure 4: Profile of potential energy scan for the torsion angle $\mathrm{C}_{15}-$ $\mathrm{N}_{14}-\mathrm{C}_{12}-\mathrm{C}_{3}$.

near $1490 \mathrm{~cm}^{-1}$ can be very weak. The fifth ring stretching vibration is active near $1315 \pm 65 \mathrm{~cm}^{-1}$, a region that overlaps strongly with that of the $\mathrm{CH}$ in-plane deformation. The sixth ring stretching vibration, or the ring breathing mode, appears as a weak band near $1000 \mathrm{~cm}^{-1}$, in mono-, 1, 3di-, and 1, 3, 5-trisubstituted benzenes. In the otherwise substituted benzenes, however, this vibration is substituent sensitive and difficult to distinguish from the ring in-plane deformation $[21,36]$.

The $v \mathrm{Ph}$ modes are expected in the region 1285$1610 \mathrm{~cm}^{-1}$ and $1280-1630 \mathrm{~cm}^{-1}$ for PhI and PhII rings, respectively [21]. The DFT calculations give the Ph stretching modes in the range $1290-1598 \mathrm{~cm}^{-1}$ and $1265-1578 \mathrm{~cm}^{-1}$ for PhI and PhII, respectively. For the title compound the phenyl ring stretching modes are observed at 1567, 1500 (IR), $1581,1486 \mathrm{~cm}^{-1}$ (Raman) and 1587, 1528, 1403, $1130 \mathrm{~cm}^{-1}$ (IR), $1133 \mathrm{~cm}^{-1}$ (Raman), for PhI and PhII, respectively. Most of the modes are not pure but contain significant contributions from other modes also. The ring breathing mode of the parasubstituted benzenes with entirely different substituents [36] has been reported in the interval 780$880 \mathrm{~cm}^{-1}$. For the title $\mathrm{c}$ ompound, the ring breathing mode $\mathrm{PhII}$ is observed at $757 \mathrm{~cm}^{-1}$ in the IR spectrum which is supported by the computational result at $747 \mathrm{~cm}^{-1}$. The ring breathing mode of $\mathrm{PhI}$ is observed at $1001 \mathrm{~cm}^{-1}$ in the Raman spectrum and the theoretical calculated value is 


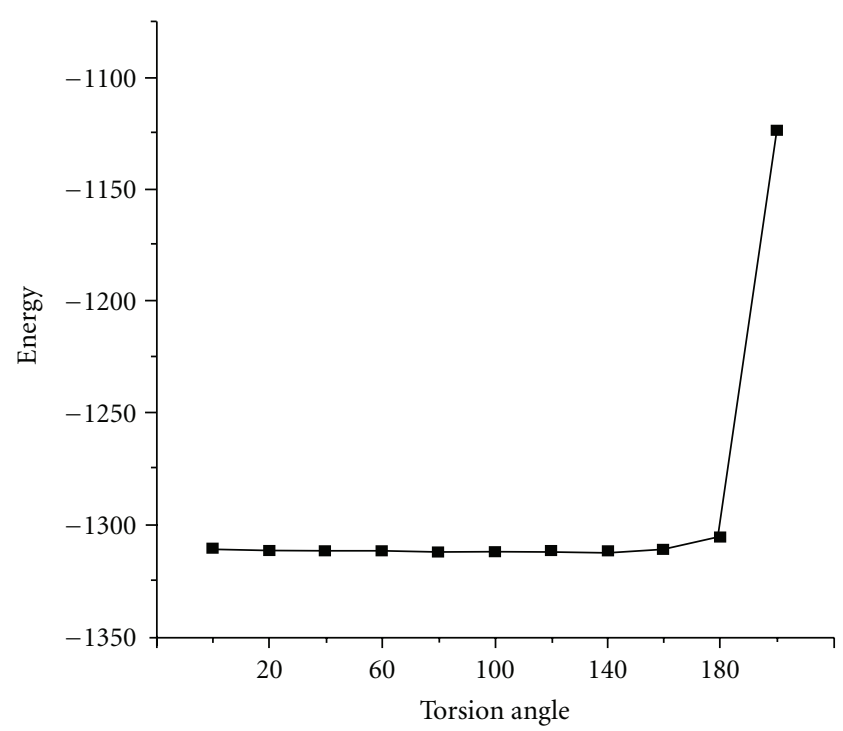

Figure 5: Profile of potential energy scan for the torsion angle $\mathrm{N}_{28}-$ $\mathrm{S}_{25}-\mathrm{C}_{22}-\mathrm{C}_{20}$.

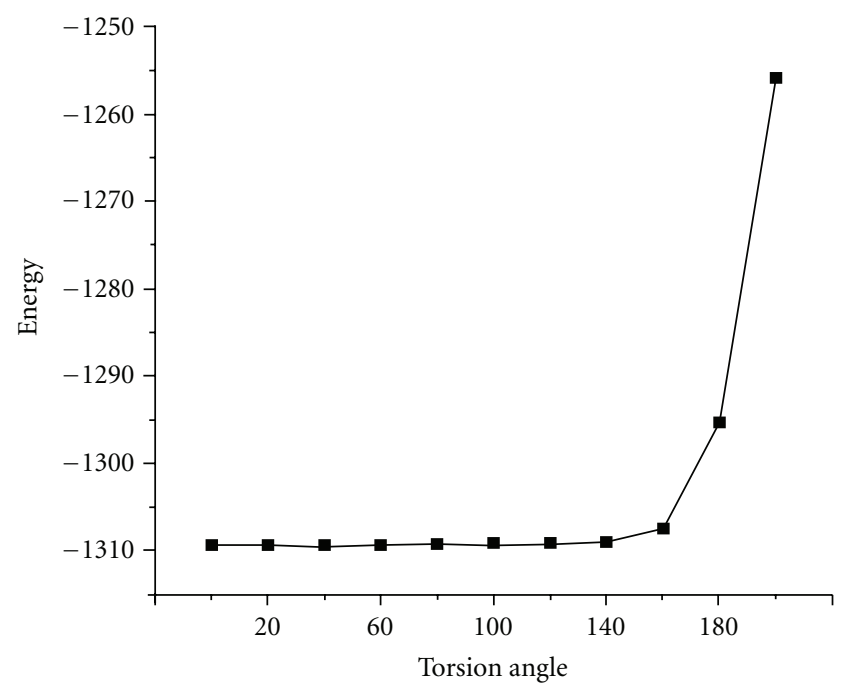

FIGURE 6: Profile of potential energy scan for the torsion angle $\mathrm{N}_{31}-$ $\mathrm{C}_{30}-\mathrm{N}_{28}-\mathrm{S}_{25}$.

$990 \mathrm{~cm}^{-1}$, as expected [21]. The in-plane and out-of-plane $\mathrm{CH}$ deformation bands of the phenyl ring are expected above and below $1000 \mathrm{~cm}^{-1}$ [21]. The in-plane $\mathrm{CH}$ deformation bands are assigned at 1319, 1160, 1156, 1096, 1076, and $1013 \mathrm{~cm}^{-1}$ for PhI and at 1317, 1230, 1103, and $1044 \mathrm{~cm}^{-1}$ for PhII theoretically. The strong $\gamma \mathrm{CH}$ occurring at $840 \pm$ $50 \mathrm{~cm}^{-1}$ is typical for 1,4 -disubstituted benzenes and the band observed at $804 \mathrm{~cm}^{-1}$ in the IR spectrum is assigned to this mode [21]. The in-plane and out-of-plane deformation modes of the phenyl ring are also identified and assigned (Table 2).

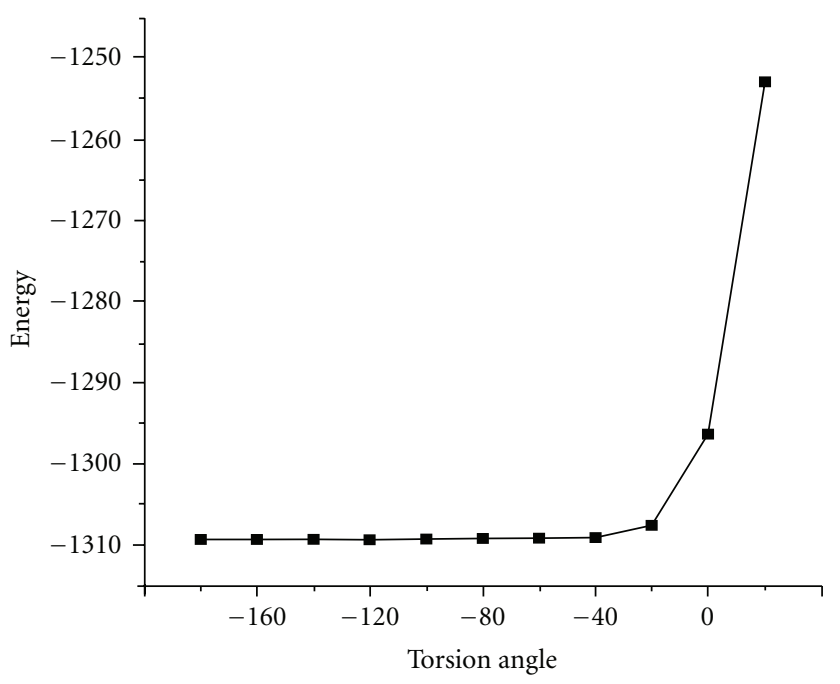

FIGURe 7: Profile of potential energy scan for the torsion angle $\mathrm{N}_{32}-$ $\mathrm{C}_{30}-\mathrm{N}_{28}-\mathrm{S}_{25}$.

The IR bands in the region $2882-1915 \mathrm{~cm}^{-1}$ and their large broadening support the intramolecular hydrogen bonding [37].

4.2. Geometrical Parameters and First Hyperpolarizability. To the best of our knowledge, no X-ray crystallographic data of this molecule has yet been established. However, the theoretical results obtained are almost comparable with the reported structural parameters of the parent molecules.

Loughrey et al. [38] reported the bond lengths, $\mathrm{S}_{25}-$ $\mathrm{O}_{27}=1.4337, \mathrm{~S}_{25}-\mathrm{O}_{26}=1.4256, \mathrm{~S}_{25}-\mathrm{N}_{28}=1.6051, \mathrm{~S}_{25}-$ $\mathrm{C}_{22}=1.7737, \mathrm{C}_{15}-\mathrm{N}_{14}=1.4212, \mathrm{C}_{12}-\mathrm{N}_{14}=1.2712$, whereas the corresponding values for the title compound are 1.4570, $1.4658,1.7054,1.7883,1.4020$, and $1.2816 \AA$. For the title compound, the DFT calculations give the bond angles, $\mathrm{C}_{22}-$ $\mathrm{S}_{25}-\mathrm{O}_{27}=108.9, \mathrm{C}_{22}-\mathrm{S}_{25}-\mathrm{O}_{26}=107.7, \mathrm{~S}_{25}-\mathrm{N}_{28}-\mathrm{H}_{29}=112.2$, $\mathrm{O}_{27}-\mathrm{S}_{25}-\mathrm{O}_{26}=122.2, \mathrm{O}_{27}-\mathrm{S}_{25}-\mathrm{N}_{28}=108.0, \mathrm{O}_{26}-\mathrm{S}_{25}-\mathrm{N}_{28}=$ $101.5, \mathrm{~N}_{28}-\mathrm{S}_{25}-\mathrm{C}_{22}=107.7, \mathrm{C}_{15}-\mathrm{N}_{14}-\mathrm{C}_{12}=120.1, \mathrm{~S}_{25}-\mathrm{C}_{22}-$ $\mathrm{C}_{18}=118.7, \mathrm{~S}_{25}-\mathrm{C}_{22}-\mathrm{C}_{20}=120.1, \mathrm{C}_{22}-\mathrm{C}_{18}-\mathrm{C}_{16}=119.3, \mathrm{C}_{18}-$ $\mathrm{C}_{16}-\mathrm{C}_{15}=120.5, \mathrm{C}_{16}-\mathrm{C}_{15}-\mathrm{C}_{17}=119.2, \mathrm{~N}_{14}-\mathrm{C}_{15}-\mathrm{C}_{17}=123.0$, $\mathrm{N}_{14}-\mathrm{C}_{15}-\mathrm{C}_{16}=117.8, \mathrm{C}_{15}-\mathrm{C}_{17}-\mathrm{C}_{20}=120.7, \mathrm{~N}_{14}-\mathrm{C}_{12}-\mathrm{C}_{3}=$ $122.8^{\circ}$, whereas the corresponding reported values are 106.5, $107.4,110.0,119.5,106.1,107.7,109.3,118.8,120.5,119.1$, $120.4,119.9,120.3,119.3,122.4,118.2,120.4$, and $124.4^{\circ}$ [38].

Loughrey et al. [38] reported the torsion angles, $\mathrm{O}_{27}-$ $\mathrm{S}_{25}-\mathrm{C}_{22}-\mathrm{C}_{18}=-142.0, \mathrm{O}_{27}-\mathrm{S}_{25}-\mathrm{C}_{22}-\mathrm{C}_{20}=38.5, \mathrm{O}_{26}-\mathrm{S}_{25}-$ $\mathrm{C}_{22}-\mathrm{C}_{18}=-12.8, \mathrm{O}_{26}-\mathrm{S}_{25}-\mathrm{C}_{22}-\mathrm{C}_{20}=167.7, \mathrm{~N}_{28}-\mathrm{S}_{25}-\mathrm{C}_{22}-$ $\mathrm{C}_{18}=103.8, \mathrm{~N}_{28}-\mathrm{S}_{25}-\mathrm{C}_{22}-\mathrm{C}_{20}=-75.7, \mathrm{C}_{12}-\mathrm{N}_{14}-\mathrm{C}_{15}-\mathrm{C}_{16}=$ 143.9, $\mathrm{C}_{12}-\mathrm{N}_{14}-\mathrm{C}_{15}-\mathrm{C}_{17}=-39.2, \mathrm{C}_{15}-\mathrm{N}_{14}-\mathrm{C}_{12}-\mathrm{C}_{3}=117.6$, $\mathrm{S}_{25}-\mathrm{C}_{22}-\mathrm{C}_{18}-\mathrm{C}_{16}=-179.9, \mathrm{C}_{20}-\mathrm{C}_{22}-\mathrm{C}_{18}-\mathrm{C}_{16}=-0.4, \mathrm{~S}_{25}-$ $\mathrm{C}_{22}-\mathrm{C}_{20}-\mathrm{C}_{17}=179.2, \mathrm{C}_{18}-\mathrm{C}_{16}-\mathrm{C}_{15}-\mathrm{N}_{14}=178.1, \mathrm{~N}_{14}-\mathrm{C}_{15}-$ $\mathrm{C}_{17}-\mathrm{C}_{20}=-178.6, \mathrm{~N}_{14}-\mathrm{C}_{12}-\mathrm{C}_{3}-\mathrm{C}_{2}=8.1, \mathrm{~N}_{14}-\mathrm{C}_{12}-\mathrm{C}_{3}-\mathrm{C}_{4}=$ $-172.6^{\circ}$. For the title compound, the corresponding torsion angles are $148.1,-30.8,13.7,-165.2,-95.1,86.0,-140.4$, 
TABle 2: Calculated vibrational wavenumbers (scaled), measured infrared and Raman band positions, and assignments of (E)-4(benzylideneamino)-N-carbamimidoyl benzenesulfonamide.

\begin{tabular}{|c|c|c|c|c|c|}
\hline $\begin{array}{l}\mathrm{HF} \\
v\left(\mathrm{~cm}^{-1}\right)\end{array}$ & $\begin{array}{l}\text { B3PW91 } \\
v\left(\mathrm{~cm}^{-1}\right)\end{array}$ & $\begin{array}{c}\text { B3LYP } \\
v\left(\mathrm{~cm}^{-1}\right)\end{array}$ & $v_{(\mathrm{IR})}$ & $v_{\text {(Raman) }}$ & Assignments \\
\hline 3563 & 3532 & 3501 & - & - & $v_{\mathrm{as}} \mathrm{NH}_{2}(100)$ \\
\hline 3450 & 3476 & 3453 & 3459 & - & $v \mathrm{~N}_{31} \mathrm{H}_{35}$ \\
\hline 3443 & 3426 & 3399 & 3421 & - & $v_{\mathrm{s}} \mathrm{NH}_{2}$ \\
\hline 3376 & 3381 & 3351 & 3351,3222 & - & $v \mathrm{~N}_{28} \mathrm{H}_{29}(100)$ \\
\hline 3072 & 3136 & 3132 & 3073 & - & $v$ CH II (97) \\
\hline 3052 & 3115 & 3109 & - & - & vCH II (99) \\
\hline 3042 & 3107 & 3100 & - & - & $v \mathrm{CH}$ I (96) \\
\hline 3037 & 3098 & 3090 & - & - & vCH II (99) \\
\hline 3027 & 3098 & 3087 & - & - & $v \mathrm{CH}$ I (94) \\
\hline 3025 & 3089 & 3082 & - & - & $v \mathrm{CH}$ II (97) \\
\hline 3014 & 3087 & 3076 & - & - & $v \mathrm{CH}$ I (94) \\
\hline 3001 & 3076 & 3066 & - & 3066 & $v \mathrm{CH}$ I (93) \\
\hline 2991 & 3067 & 3057 & 3026 & - & $v \mathrm{CH}$ I (93) \\
\hline 2895 & 2923 & 2915 & - & - & $v \mathrm{C}_{12} \mathrm{H}_{13}$ (99) \\
\hline 1683 & 1637 & 1687 & - & - & $v \mathrm{C}_{12} \mathrm{~N}_{14}$ \\
\hline 1676 & 1655 & 1641 & - & - & $v \mathrm{C}_{30} \mathrm{~N}_{31}(60)$ \\
\hline 1640 & 1609 & 1602 & 1620 & 1625 & $\delta \mathrm{NH}_{2}(87)$ \\
\hline 1617 & 1603 & 1598 & - & - & $v \mathrm{Ph} \mathrm{I}(53)$ \\
\hline 1600 & 1590 & 1578 & 1587 & - & $v \mathrm{Ph}$ II (58) \\
\hline 1590 & 1583 & 1571 & 1567 & 1581 & $v \mathrm{Ph} \mathrm{I}(63)$ \\
\hline 1578 & 1564 & 1552 & 1528 & - & $v \mathrm{Ph}$ II (68) \\
\hline 1501 & 1487 & 1485 & 1500 & 1486 & $v \mathrm{Ph}$ I (42), $\delta \mathrm{NH} \mathrm{I}(39)$ \\
\hline 1495 & 1473 & 1472 & - & - & $v \mathrm{Ph}$ II (60), $\delta \mathrm{CH} \mathrm{I}(15), \delta \mathrm{CH}$ II $(20)$ \\
\hline 1472 & 1442 & 1442 & 1450 & 1446 & $\delta \mathrm{NH}(65)$ \\
\hline 1456 & 1440 & 1430 & - & - & $v \mathrm{Ph} \mathrm{I}(64), \delta \mathrm{CH} \mathrm{I}(34)$ \\
\hline 1407 & 1397 & 1394 & 1403 & - & $v \mathrm{Ph}$ II (57), $\delta \mathrm{CH}$ II (36) \\
\hline 1387 & 1370 & 1371 & 1358 & 1360 & $v_{\mathrm{as}} \mathrm{SO}_{2}(60), \delta \mathrm{CH}_{13}(28)$ \\
\hline 1334 & 1335 & 1319 & - & - & $\delta \mathrm{CH} \mathrm{I}(68)$ \\
\hline 1319 & 1332 & 1317 & 1313 & 1310 & $\delta \mathrm{CH}$ II (84) \\
\hline 1310 & 1314 & 1299 & - & - & $\delta \mathrm{NH}(56)$ \\
\hline 1242 & 1294 & 1290 & - & - & $v \mathrm{Ph} \mathrm{I}(67), \delta \mathrm{CH} \mathrm{I}(17)$ \\
\hline 1233 & 1279 & 1281 & - & - & $v \mathrm{Ph} \mathrm{I}(40), v \mathrm{C}_{15} \mathrm{~N}_{14}(58)$ \\
\hline 1215 & 1272 & 1265 & - & - & $v \mathrm{Ph}$ II $(82)$ \\
\hline 1185 & 1236 & 1230 & 1236 & 1234 & $v \mathrm{Ph}$ II (14), $\delta \mathrm{CH}$ II (42) \\
\hline 1181 & 1187 & 1181 & 1182 & 1188 & $\delta \mathrm{CH} \mathrm{I}(36), \delta \mathrm{NH}_{2}(56)$ \\
\hline 1172 & 1159 & 1160 & 1170 & 1168 & $v \mathrm{C}_{15} \mathrm{~N}_{14}(18), \delta \mathrm{CH}$ II $(14), \delta \mathrm{CH} \mathrm{I}(60)$ \\
\hline 1148 & 1156 & 1156 & - & - & $\delta \mathrm{CH} \mathrm{I}(48), v \mathrm{Ph} \mathrm{I}(40)$ \\
\hline 1107 & 1147 & 1148 & 1130 & 1133 & $v_{\mathrm{s}} \mathrm{SO}_{2}(46), v \mathrm{Ph}$ II (46) \\
\hline 1086 & 1118 & 1103 & - & - & $\delta \mathrm{CH}$ II $(61), \delta \mathrm{NH}_{2}(28)$ \\
\hline 1078 & 1097 & 1100 & - & - & $v \mathrm{C}_{30} \mathrm{~N}_{28}(45), \delta \mathrm{NH}_{2}$ \\
\hline 1074 & 1095 & 1096 & 1087 & 1088 & $\delta \mathrm{CH} \mathrm{I}(61), v \mathrm{Ph} \mathrm{I}(38)$ \\
\hline 1066 & 1083 & 1076 & - & - & $\delta \mathrm{CH}$ I $(73)$ \\
\hline 1058 & 1071 & 1069 & 1057 & 1065 & $v \mathrm{Ph}$ II (46) \\
\hline 1045 & 1056 & 1044 & - & - & $\delta \mathrm{CH}$ II (70) \\
\hline 1037 & 1017 & 1013 & 1010 & 1015 & $\delta \mathrm{CH} \mathrm{I}(63)$ \\
\hline 1035 & 990 & 990 & - & 1001 & $v \mathrm{Ph} \mathrm{I}$ \\
\hline 1029 & 984 & 980 & - & - & $\gamma \mathrm{CH}$ II (69) \\
\hline 1020 & 980 & 978 & 971 & 974 & $\gamma \mathrm{CH} \mathrm{I}(82)$ \\
\hline 1007 & 977 & 972 & - & - & $v \mathrm{Ph}$ II (81) \\
\hline
\end{tabular}


Table 2: Continued.

\begin{tabular}{|c|c|c|c|c|c|}
\hline $\begin{array}{l}\mathrm{HF} \\
v\left(\mathrm{~cm}^{-1}\right)\end{array}$ & $\begin{array}{l}\text { B3PW91 } \\
v\left(\mathrm{~cm}^{-1}\right)\end{array}$ & $\begin{array}{c}\text { B3LYP } \\
v\left(\mathrm{~cm}^{-1}\right)\end{array}$ & $v_{(\mathrm{IR})}$ & $v_{(\text {Raman })}$ & Assignments \\
\hline 994 & 966 & 965 & - & - & $\gamma \mathrm{CH} \mathrm{I}(80)$ \\
\hline 990 & 945 & 942 & 948 & - & $v \mathrm{C}_{30} \mathrm{~N}_{32}(68)$ \\
\hline 975 & 940 & 940 & - & - & $\gamma \mathrm{CH} \mathrm{I}(74)$ \\
\hline 897 & 925 & 926 & 921 & 922 & $\gamma \mathrm{CH}$ II (79) \\
\hline 887 & 902 & 901 & - & - & $\gamma \mathrm{CH}$ I (99) \\
\hline 873 & 867 & 865 & 884 & 882 & $\gamma \mathrm{CH}$ II (89) \\
\hline 859 & 831 & 831 & 841 & - & $v \mathrm{SN}(67)$ \\
\hline 847 & 830 & 823 & 829 & 820 & $\gamma \mathrm{CH}$ II (59), $\gamma \mathrm{CH}_{13}(15)$ \\
\hline 809 & 819 & 819 & - & - & $\gamma \mathrm{CH}$ II (57), $v \mathrm{Ph}$ I (12) \\
\hline 795 & 811 & 806 & - & - & $\delta \mathrm{NH}(82)$ \\
\hline 789 & 800 & 802 & 796 & - & $\gamma \mathrm{CH} \mathrm{I}(63)$ \\
\hline 782 & 776 & 778 & - & - & $\delta \mathrm{NH}_{2}(61)$ \\
\hline 748 & 745 & 747 & 757 & 751 & $v \mathrm{Ph}$ II (64) \\
\hline 715 & 707 & 706 & 727 & 729 & $\gamma \mathrm{NH}(69)$ \\
\hline 710 & 690 & 689 & 709 & 709 & $\gamma \mathrm{CH} \mathrm{I}(25), \delta \mathrm{Ph} \mathrm{I}(64)$ \\
\hline 694 & 678 & 679 & 692 & - & $v \mathrm{CS}(58), \delta \mathrm{CN}(23)$ \\
\hline 656 & 673 & 673 & - & - & $\delta \mathrm{SO}_{2}(40), \delta \mathrm{Ph} \mathrm{II}(18)$ \\
\hline 636 & 619 & 621 & 633 & 629 & $\delta \mathrm{Ph}$ II $(79)$ \\
\hline 626 & 612 & 610 & 612 & 610 & $\delta \mathrm{Ph} \mathrm{I}(85)$ \\
\hline 602 & 605 & 608 & - & - & $\gamma \mathrm{NH}(65)$ \\
\hline 570 & 590 & 590 & - & - & $\delta \mathrm{PhI}(29), \delta \mathrm{PhII}(20), \delta \mathrm{CN}(24)$ \\
\hline 554 & 575 & 579 & - & - & $\tau$ PhII (30), $\gamma \mathrm{CS}(17),=\mathrm{CN}(17)$ \\
\hline 521 & 542 & 541 & 556 & 543 & $\tau \operatorname{PhI}(51), \delta \mathrm{CC}_{12}(31)$ \\
\hline 510 & 530 & 529 & - & - & $\delta \mathrm{NH}(46), \delta \mathrm{NH}_{2}(32)$ \\
\hline 478 & 509 & 510 & 515 & - & $\delta \mathrm{NH}_{2}(28), \delta \mathrm{SO}_{2}(47), \delta \mathrm{CN}(14)$ \\
\hline 468 & 497 & 496 & - & - & $\gamma \mathrm{NH}_{2}(41), \tau \mathrm{PhI}(20)$ \\
\hline 442 & 455 & 457 & 469 & 460 & $\delta \mathrm{SO}_{2}(41), \gamma \mathrm{NH}(39)$ \\
\hline 427 & 424 & 419 & 435 & - & $\tau$ PhII $(84)$ \\
\hline 419 & 405 & 407 & 408 & 415 & $\tau \mathrm{PhI}(84)$ \\
\hline 413 & 399 & 401 & - & - & $\delta \mathrm{SO}_{2}(40) \delta \mathrm{PhII}(31)$ \\
\hline 376 & 398 & 397 & - & - & $\delta \mathrm{CN}(35), \tau$ PhII $(33)$ \\
\hline 345 & 366 & 365 & - & - & $\delta \mathrm{SO}_{2}(47), \delta \mathrm{CS}(24)$ \\
\hline 320 & 355 & 353 & - & - & $\delta \mathrm{SO}_{2}(39), \tau \mathrm{CC}(25)$ \\
\hline 299 & 349 & 347 & - & 343 & $\gamma \mathrm{NH}(64), \delta \mathrm{SO}_{2}(15)$ \\
\hline 275 & 342 & 338 & - & - & $\tau \mathrm{NH}(39), \tau \mathrm{PhI}(36)$ \\
\hline 270 & 299 & 298 & - & 295 & $\delta \mathrm{SO}_{2}(40), \tau \mathrm{NH}(23)$ \\
\hline 247 & 275 & 275 & - & - & $\delta \mathrm{SO}_{2}(50), \gamma \mathrm{NH}(28)$ \\
\hline 243 & 253 & 253 & - & 255 & $\tau \mathrm{SO}_{2}(36), \delta \mathrm{CN}(31)$ \\
\hline 204 & 216 & 217 & - & 209 & $\tau \mathrm{SO}_{2}(48), \tau \mathrm{PhII}(29)$ \\
\hline 162 & 177 & 179 & - & - & $\tau \mathrm{SO}_{2}(28), \tau \mathrm{NH}(21), \tau \mathrm{CS}(17)$ \\
\hline 156 & 160 & 160 & - & - & $\delta \mathrm{NH}(47), \tau \mathrm{CN}(26)$ \\
\hline 133 & 138 & 138 & - & - & $\delta \mathrm{NH}(44), \delta \mathrm{SO}_{2}(25)$ \\
\hline 120 & 127 & 128 & - & 120 & $\tau \mathrm{CN}(45), \delta \mathrm{SO}_{2}(38)$ \\
\hline 108 & 107 & 107 & - & - & $\tau \mathrm{NH}(34), \tau \mathrm{CN}(27)$ \\
\hline 80 & 82 & 81 & - & - & $\tau \mathrm{CN}(38), \tau \mathrm{SO}_{2}$ \\
\hline 59 & 55 & 55 & - & - & $\tau \mathrm{CN}(38), \delta \mathrm{SO}_{2}(29)$ \\
\hline 49 & 48 & 48 & - & - & $\tau \mathrm{SO}_{2}(25), \tau \mathrm{CN}(34)$ \\
\hline 41 & 43 & 42 & - & - & $\tau \mathrm{CN}(53), \tau \mathrm{SO}_{2}(12)$ \\
\hline 25 & 24 & 24 & - & - & $\tau \mathrm{Ph}$ II (63), $\tau \mathrm{CN}(56), \tau \mathrm{SO}_{2}(21)$ \\
\hline 22 & 22 & 23 & 一 & 一 & $\tau \mathrm{SO}_{2}(39), \tau \mathrm{CN}(24), \tau \mathrm{NH}(10)$ \\
\hline
\end{tabular}

$v$ : stretching, $\delta$ : in-plane bending, $\gamma$ : out-of-plane bending, $\tau$ : torsion, PhI: monosubstituted phenyl ring, PhII: parasubstituted phenyl ring. Subscripts: as: asymmetric; s: symmetric. 
$42.1,-177.0,-179.4,-0.4,178.5,-179.6,178.6,1.1$, and $-179.0^{\circ}$.

Petrov et al. [39] reported the molecular structure and conformations of benzenesulfonamide by gas electron diffraction and quantum chemical calculations and according to their results, the bond lengths, $\mathrm{CS}, \mathrm{SN}, \mathrm{SO}$ vary in the range 1.7756-1.7930, 1.6630-1.6925, 1.4284-1.4450 the bond angles, CSN, CSO, NSO, HNS, HNH vary in the range, 103.9-107.1, 107.6-107.8, 105.5-107.7, 111.0$113.7,112.6-113.6 \AA$. These values are in agreement with the corresponding values for the title compound.

Lasibal et al. [40] reported the bond lengths $\mathrm{SO}=1.4269$ $1.4291, \mathrm{SN}=1.6202, \mathrm{SC}=1.7582, \mathrm{~N}_{32}-\mathrm{C}_{30}=1.4103, \mathrm{~N}_{31}-\mathrm{C}_{30}$ $=1.2723, \mathrm{~N}_{28}-\mathrm{C}_{30}=1.3483 \AA$, whereas the corresponding values in the present case are 1.4658-1.4570, 1.7054, 1.7833, $1.3930,1.2764$, and $1.4033 \AA$. The values of bond angles $\mathrm{O}_{27}-\mathrm{S}_{25}-\mathrm{O}_{26}=118.6, \mathrm{O}_{26}-\mathrm{S}_{25}-\mathrm{N}_{28}=108.9, \mathrm{O}_{27}-\mathrm{S}_{25}-\mathrm{N}_{28}=$ 104.9, $\mathrm{O}_{27,26}-\mathrm{S}_{25}-\mathrm{C}_{22}=107.9-108.3, \mathrm{~N}_{28}-\mathrm{S}_{25}-\mathrm{C}_{22}=107.9$, $\mathrm{C}_{30}-\mathrm{N}_{28}-\mathrm{S}_{25}=123.0^{\circ}$ reported by Lasibal et al. [40] are in agreement with our values.

At $\mathrm{C}_{3}$ position, the bond angles $\mathrm{C}_{4}-\mathrm{C}_{3}-\mathrm{C}_{2}, \mathrm{C}_{4}-\mathrm{C}_{3}-\mathrm{C}_{12}$, and $C_{2}-C_{3}-C_{12}$ are $119.2,119.3$, and $121.5^{\circ}$ respectively. This asymmetry in angles reveals the interaction between azomethane and the phenyl groups. At $\mathrm{C}_{15}$ position the angles $\mathrm{C}_{17}-\mathrm{C}_{15}-\mathrm{N}_{14}$ is increased by $3^{\circ}$ and $\mathrm{C}_{16}-\mathrm{C}_{15}-\mathrm{N}_{14}$ is reduced by $2.2^{\circ}$ from $120^{\circ}$ which reveals the interaction between $\mathrm{N}_{14}$ and $\mathrm{H}_{21}$ atoms. At $\mathrm{C}_{22}$ position $\mathrm{C}_{20}-\mathrm{C}_{22}-\mathrm{S}_{25}=120.1^{\circ}$ and $\mathrm{C}_{20}-\mathrm{C}_{22}-\mathrm{C}_{18}=121.2^{\circ}$ which shows the interaction between $\mathrm{SO}_{2}$ group with $\mathrm{H}_{24}$ atom. At $\mathrm{N}_{28}$ position, $\mathrm{S}_{25}-\mathrm{N}_{28}-\mathrm{H}_{29}$ is reduced by $7.8^{\circ}$ and $\mathrm{S}_{25}-\mathrm{N}_{28}-\mathrm{C}_{30}$ is increased by $6.8^{\circ}$ from $120^{\circ}$ which shows the interaction between $\mathrm{H}_{29}$ and $\mathrm{O}_{27}$. The $\mathrm{C}_{12}=\mathrm{N}_{14}$ moiety is slightly tilted from monosubstituted phenyl ring as is evident from the torsion angles $\mathrm{C}_{5}-\mathrm{C}_{4}-\mathrm{C}_{3}-$ $\mathrm{C}_{12}=-179.8, \mathrm{C}_{4}-\mathrm{C}_{3}-\mathrm{C}_{12}-\mathrm{N}_{14}=-179.0, \mathrm{C}_{1}-\mathrm{C}_{2}-\mathrm{C}_{3}-\mathrm{C}_{12}=$ $179.9, \mathrm{C}_{2}-\mathrm{C}_{3}-\mathrm{C}_{12}-\mathrm{N}_{14}=177.4^{\circ}$ and is more tilted from the para substituted phenyl ring as is evident from the torsion angles $\mathrm{C}_{20}-\mathrm{C}_{17}-\mathrm{C}_{15}-\mathrm{N}_{14}=178.6, \mathrm{C}_{17}-\mathrm{C}_{15}-\mathrm{C}_{14}-\mathrm{C}_{12}=42.1$, $\mathrm{C}_{18}-\mathrm{C}_{16}-\mathrm{C}_{15}-\mathrm{N}_{14}=179.6$, and $\mathrm{C}_{16}-\mathrm{C}_{15}-\mathrm{N}_{14}-\mathrm{C}_{12}=-140.4^{\circ}$. The torsion angle $\mathrm{S}_{25}-\mathrm{N}_{28}-\mathrm{C}_{30}-\mathrm{N}_{32}=-169.9$ and $\mathrm{S}_{25}-\mathrm{N}_{28}-$ $\mathrm{C}_{30}-\mathrm{N}_{31}=12.6^{\circ}$, which shows that the $\mathrm{N}_{31}$ and $\mathrm{N}_{32}$ atoms are in different planes.

Nonlinear optics deal with the interaction of applied electromagnetic fields in various materials to generate new electromagnetic fields, altered in wavenumber, phase, or other physical properties [41]. Organic molecules able to manipulate photonic signals efficiently are of importance in technologies such as optical communication, optical computing, and dynamic image processing $[42,43]$. In this context, the dynamic first hyperpolarizability of the title compound is also calculated in the present study. The first hyperpolarizability $\left(\beta_{0}\right)$ of this novel molecular system is calculated using B3LYP method, based on the finite field approach. In the presence of an applied electric field, the energy of a system is a function of the electric field. First hyperpolarizability is a third rank tensor that can be described by a $3 \times 3 \times 3$ matrix. The 27 components of the $3 \mathrm{D}$ matrix can be reduced to 10 components due to the Kleinman symmetry [44]. The components of $\beta$ are defined as the coefficients in the Taylor series expansion of the energy in the external electric field. When the electric field is weak and homogeneous, this expansion becomes

$$
\begin{aligned}
E= & E_{0}-\sum_{i} \mu_{i} F_{i}-\frac{1}{2} \sum_{i j} \alpha_{i j} F^{i} F^{j}-\frac{1}{6} \sum_{i j k} \beta_{i j k} F^{i} F^{j} F^{k} \\
& -\frac{1}{24} \sum_{i j k l} \gamma_{i j k l} F^{i} F^{j} F^{k} F^{l}+\cdots,
\end{aligned}
$$

where $E_{0}$ is the energy of the unperturbed molecule, $F^{i}$ is the field at the origin, $\mu_{i}, \alpha_{i j}, \beta_{i j k}$, and $\gamma_{i j k l}$ are the components of dipole moment, polarizability, the first hyperpolarizabilities, and second hyperpolarizabilities, respectively. The calculated first hyperpolarizability of the title compound is $6.25 \times$ $10^{-30} \mathrm{esu}$, which is comparable with the reported values of similar derivatives, but experimental evaluation of this data is not readily available. Kucharski et al. [45] reported the first hyperpolarizability of certain sulfonamide amphiphiles by calculation and hyper-Rayleigh scattering in the range $0.2156-0.189 \times 10^{-30}$ esu. We conclude that the title compound is an attractive object for future studies of nonlinear optical properties.

\section{Conclusion}

The FT-IR and FT-Raman spectrum of the title compound were recorded and analyzed. The molecular geometry and vibrational wavenumbers were calculated using $\mathrm{HF}$ and DFT methods, and the optimized geometrical parameters (B3LYP) are in agreement with that of reported similar derivatives. The red shift of the $\mathrm{N}-\mathrm{H}$ stretching band in the infrared spectrum from the computed wavenumber indicates the weakening of the $\mathrm{N}-\mathrm{H}$ bond. The calculated first hyperpolarizability is comparable with the reported value of similar derivative and may be an attractive object for further studies of non linear optics. Potential energy surface scan studies have been carried out to understand the stability of planar and non planar structures of the molecule.

\section{References}

[1] A. K. Biswas, G. S. Rao, N. Kondaiah, A. S. R. Anjaneyulu, and J. K. Malik, "Simple multiresidue method for monitoring of trimethoprim and sulfonamide residues in Buffalo meat by high-performance liquid chromatography," Journal of Agricultural and Food Chemistry, vol. 55, no. 22, pp. 8845-8850, 2007.

[2] C. T. Supuran, "Carbonic anhydrases: novel therapeutic applications for inhibitors and activators," Nature Reviews Drug Discovery, vol. 7, no. 2, pp. 168-181, 2008.

[3] C. T. Supuran, "Diuretics: from classical carbonic anhydrase inhibitors to novel applications of the sulfonamides," Current Pharmaceutical Design, vol. 14, no. 7, pp. 641-648, 2008.

[4] C. Temperini, A. Cecchi, A. Scozzafava, and C. T. Supuran, "Carbonic anhydrase inhibitors. Comparison of chlorthalidone, indapamide, trichloromethiazide, and furosemide $\mathrm{X}$ ray crystal structures in adducts with isozyme II, when several water molecules make the difference," Bioorganic and Medicinal Chemistry, vol. 17, no. 3, pp. 1214-1221, 2009.

[5] J. Y. Winum, C. Temperini, K. El Cheikh et al., "Carbonic anhydrase inhibitors: clash with Ala65 as a means for designing inhibitors with low affinity for the ubiquitous isozyme II, 
exemplified by the crystal structure of the topiramate sulfamide analogue," Journal of Medicinal Chemistry, vol. 49, no. 24, pp. 7024-7031, 2006.

[6] Z. Guo and P. J. Sadler, "Metals in medicine," Angewandte Chemie, vol. 38, no. 11, pp. 1513-1531, 1999.

[7] R. X. Yuan, R. G. Xiong, and Z. F. Chen, "Crystal structure of zinc(II) 2-sulfanilamidopyrimidine: a widely used topical burn drug," Journal of the Chemical Society-Dalton Transactions, vol. 6, no. 774, p. 776, 2001.

[8] T. H. Maren, "Relatons between structure and biological activity of sulfonamides," Annual Review of Pharmacology and Toxicology, vol. 16, pp. 309-327, 1976.

[9] C. T. Supuran, A. Scozzafava, and A. Mastrolorenzo, "Bacterial proteases: current therapeutic use and future prospects for the development of new antibiotics," Expert Opinion on Therapeutic Patents, vol. 11, no. 2, pp. 221-259, 2001.

[10] A. E. Boyd, "Sulfonylurea receptors, ion channels, and fruit flies," Diabetes, vol. 37, no. 7, pp. 847-850, 1988.

[11] T. Owa and T. Nagasu, "Novel sulphonamide derivatives for the treatment of cancer," Expert Opinion on Therapeutic Patents, vol. 10, no. 11, pp. 1725-1740, 2000.

[12] C. W. Thornber, "Isosterism and molecular modification in drug design," Chemical Society Reviews, vol. 8, no. 4, pp. 563$580,1979$.

[13] U. K. Singh, S. N. Pandeya, A. Singh, B. K. Srivastava, and M. Pandey, "Synthesis and antimicrobial activity of Schiff's and $\mathrm{N}$-mannich bases of isatin and its derivatives with 4-amino-Ncarbamimidoyl benzene sulfonamide," International Journal of Pharmaceutical Sciences and Drug Research, vol. 2, no. 2, pp. 151-154, 2010.

[14] X. Liu, L. Liu, X. Lu, J. Zheng, W. Wang, and Y. Fang, "Interference second harmonic generation investigation of the chromophore orientation of hemicyanine dye molecules in a Langmuir-Blodgett monolayer," Thin Solid Films, vol. 217, no. 1-2, pp. 174-177, 1992.

[15] Y. Miyamoto, K. Kaifu, T. Koyano, M. Saito, and M. Kato, "Second harmonic generation from mixed Langmuir-Blodgett films of N-acyl-p-nitroaniline and its homologous amphiphile," Thin Solid Films, vol. 210-211, pp. 178-181, 1992.

[16] M. J. Frisch, G. W. Trucks, H. B. Schlegel et al., Gaussian 03, Revision C.02, Gaussian, Inc, Wallingford, Conn, USA, 2004.

[17] J. B. Foresman, Expploring Chemistry with Electronic Structure Methods: A Guide to Using Gaussian, Gaussian Inc., Pittsburgh, Pa, USA, 1996.

[18] J. M. L. Martin and C. Van Alsenoy, GAR2PED, A Program to obtain a Potential Energy Distribution from a Gaussian Archive Record, University of Antwerp, Brussels, Belgium, 2007.

[19] P. Flukiger, H. P. Luthi, S. Portmann, and J. Weber, MOLEKEL 4.3, Swiss Centre for Scientific Computing, Manno, Lugano, Switzerland, 2002.

[20] S. Portmann and H. P. Lüthi, "MOLEKEL: an interactive molecular graphics tool," Chimia, vol. 54, no. 12, pp. 766-769, 2000.

[21] N. P. G. Roges, A Guide to the Complete Interpretation of the Infrared Spectra of Organic Structures, John Wiley \& Sons, New York, NY, USA, 1994.

[22] Z. H. Chohan, M. H. Youssoufi, A. Jarrahpour, and T. B. Hadda, "Identification of antibacterial and antifungal pharmacophore sites for potent bacteria and fungi inhibition: indolenyl sulfonamide derivatives," European Journal of Medicinal Chemistry, vol. 45, no. 3, pp. 1189-1199, 2010.
[23] A. Hangen, A. Bodoki, L. Orpean, G. Alzuet, M. Liu-Gonzalez, and J. Borras, "Synthesis, crystallographic and spectroscopic characterization and magnetic properties of dimer and monomer ternary copper(II) complexes with sulfonamide derivatives and 1,10-phenanthroline. Nuclease activity by the oxidative mechanism," Polyhedron, vol. 29, no. 4, pp. 1305-1313, 2010.

[24] I. Yalcin, E. Sener, T. Ozden, S. Ozden, and A. Akin, "Synthesis and microbiological activity of 5-methyl-2-[p-substituted phenyl]benzoxazoles," European Journal of Medicinal Chemistry, vol. 25, no. 8, pp. 705-708, 1990.

[25] N. B. Colthup, L. H. Daly, and S. E. Wiberly, Introduction to Infrared and Raman Spectroscopy, Academic Press, New York, NY, USA, 1975.

[26] C. Y. Panicker, H. T. Varghese, K. C. Mariamma et al., "Spectroscopic investigations and computational study of 2-[acetyl(4-bromophenyl)carbamoyl]-4-chlorophenyl acetate," Journal of Raman Spectroscopy, vol. 41, no. 6, pp. 707-716, 2010.

[27] L. J. Bellamy, The IR Spectra of Complex Molecules, John Wiley \& Sons, New York, NY, USA, 1975.

[28] A. Spire, M. Barthes, H. Kellouai, and G. De Nunzio, "Farinfrared spectra of acetanilide revisited," Physica D, vol. 137, no. 3-4, pp. 392-401, 2000.

[29] M. Barthes, G. DeNunzio, and G. Ribet, "Polarons or proton transfer in chains of peptide groups?" Synthetic Metals, vol. 76, no. 1-3, pp. 337-340, 1996.

[30] C. Y. Panicker, K. R. Ambujakshan, H. T. Varghese et al., "FT-IR, FT-Raman and DFT calculations of 3-[(4-fluorophenyl)methylene]amino-2-phenylquinazolin-4(3H)-one," Journal of Raman Spectroscopy, vol. 40, no. 5, pp. 527-536, 2009.

[31] P. L. Anto, C. Y. Panicker, H. T. Varghese et al., "Vibrational spectroscopic studies and ab initio calculations of 5-methyl-2(P-fluorophenyl)benzoxazole," Spectrochimica Acta A, vol. 67, pp. 744-749, 2007.

[32] K. R. Ambujakshan, V. S. Madhavan, H. T. Varghese et al., "Vibrational spectroscopic studies and ab initio calcula-tions of 5-methyl-2-(p-methylaminophenyl)benzoxazole," Spectrochimica Acta A, vol. 69, no. 3, pp. 782-788, 2008.

[33] P. Sett, N. Paul, S. Chattopadhyay, and P. K. Mallick, "Molecular structures in the excited states of 2- and 4-benzylpyridine," Journal of Raman Spectroscopy, vol. 30, no. 4, pp. 277-287, 1999.

[34] J. H. S. Green, "Vibrational spectra of benzene derivativesIV. Methylphenyl sulphide, diphenyl sulphide, diphenyl disulphide and diphenyl sulphoxide," Spectrochimica Acta A, vol. 24, no. 10, pp. 1627-1637, 1968.

[35] J. Coates, "Interpretation of infrared spectra, a practical approach," in Encyclopedia of Analytical Chemistry, R. A. Meyers, Ed., John Wiley \& Sons, Chichester, UK, 2000.

[36] G. Varsanyi, Assignments of Vibrational Spectra of Seven Hundred Benzene Derivatives, John Wiley \& Sons, New York, NY, USA, 1974.

[37] D. Philip, A. John, C. Y. Panicker, and H. T. Varghese, "FTRaman, FT-IR and surface enhanced Raman scattering spectra of sodium salicylate," Spectrochimica Acta A, vol. 57, no. 8, pp. 1561-1566, 2001.

[38] B. T. Loughrey, M. L. Williams, and P. C. Healy, "4-(Benzylideneamino)benzenesulfonamide," Acta Crystallographica E, vol. 65, pp. 2087-2096, 2009.

[39] V. Petrov, V. Petrova, G. V. Girichev, H. Oberhammer, N. I. Giricheva, and S. Ivanov, "Molecular structure and conformations of benzenesulfonamide: gas electron diffraction and 
quantum chemical calculations," Journal of Organic Chemistry, vol. 71, no. 8, pp. 2952-2956, 2006.

[40] E. Labisbal, L. Rodriguez, A. Sousa-Pedrares et al., "Synthesis, characterisation and X-ray structures of diorganotin(IV) and iron(III) complexes of dianionic terdentate Schiff base ligands," Journal of Organometallic Chemistry, vol. 691, no. 7, pp. 1321-1332, 2006.

[41] Y. R. Shen, The Principles of Nonlinear Optics, John Wiley \& Sons, New York, NY, USA, 1984.

[42] P. V. Kolinsky, "New materials and their characterization for photonic device applications," Optical Engineering, vol. 31, pp. 1676-1684, 1992.

[43] D. F. Eaton, "Nonlinear optical materials," Science, vol. 253, no. 5017, pp. 281-287, 1991.

[44] D. A. Kleinman, "Nonlinear dielectric polarization in optical media," Physical Review, vol. 126, no. 6, pp. 1977-1979, 1962.

[45] S. Kucharski, R. Janik, and P. Kaatz, "First hyperpolarizability of new sulfonamide amphiphiles by calculation, and hyperRayleigh scattering," Journal of Materials Chemistry, vol. 9, no. 2, pp. 395-401, 1999. 


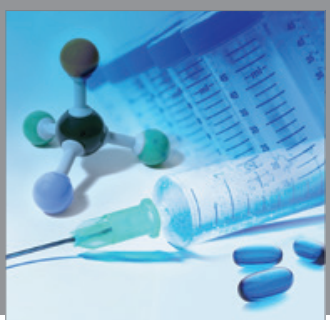

International Journal of

Medicinal Chemistry

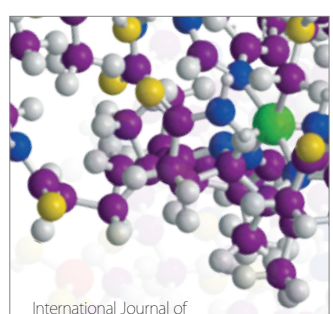

Carbohydrate Chemistry

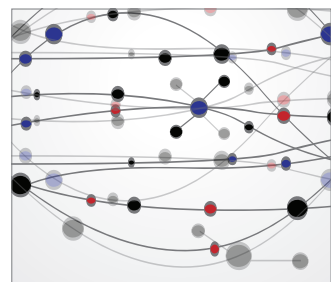

The Scientific World Journal
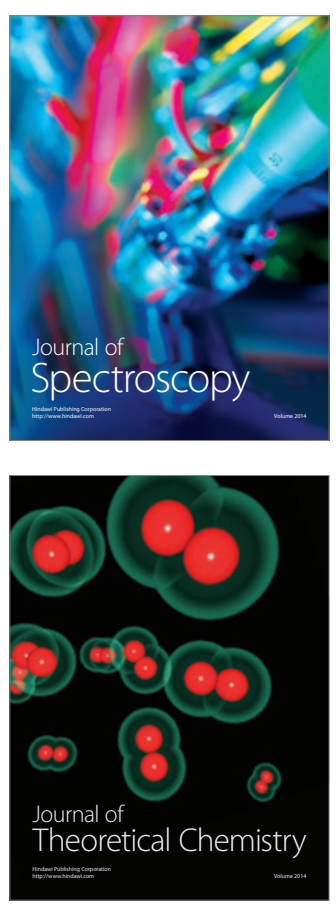
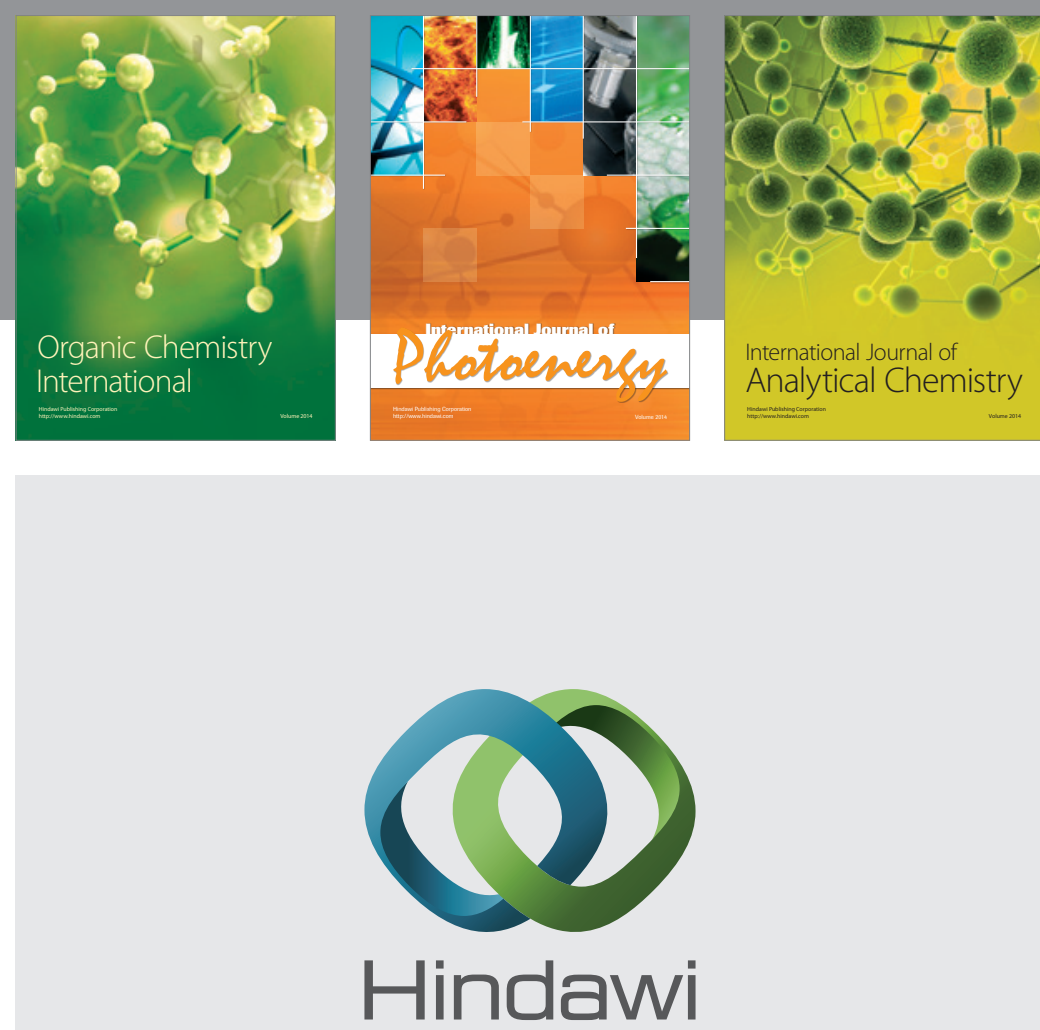

Submit your manuscripts at

http://www.hindawi.com
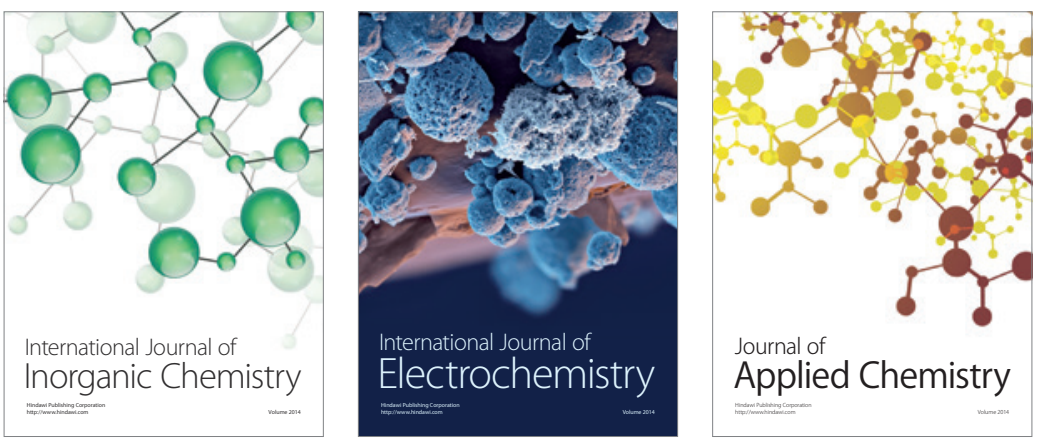

Journal of

Applied Chemistry
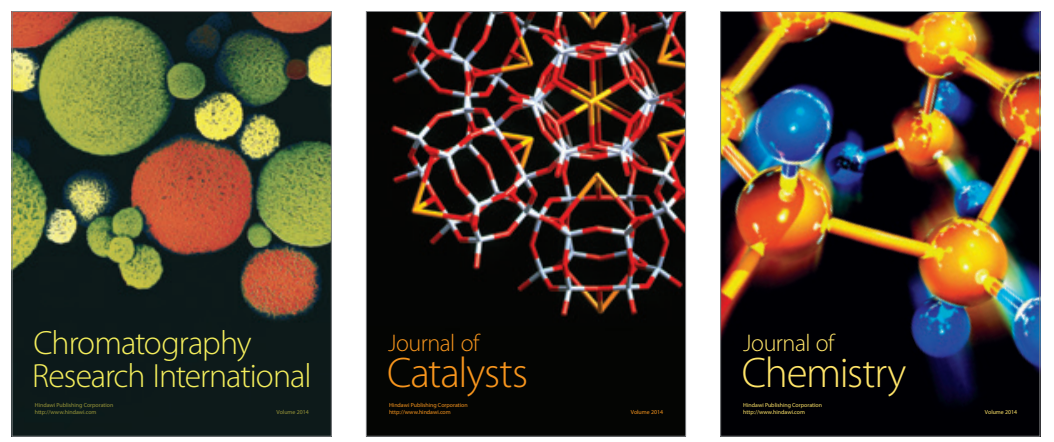
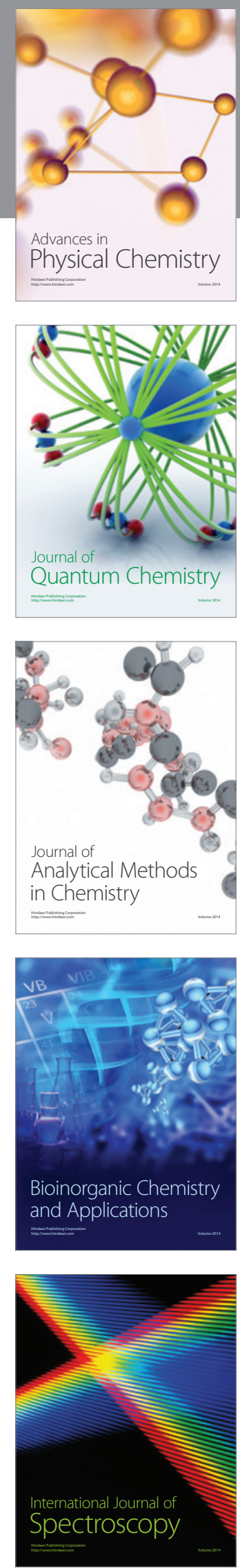